Revue de droit comparé du travail et de la sécurité sociale

3 | 2018

La participation des travailleurs dans la grande entreprise privée et publique

\title{
Le système québécois de participation des travailleurs : vers une refondation sur de nouvelles assises constitutionnelles?
}

Julie Bourgault et Michel Coutu

\section{CpenEdition}

Édition électronique

URL : https://journals.openedition.org/rdctss/1904

DOI : $10.4000 /$ rdctss. 1904

ISSN : 2262-9815

Éditeur

Centre de droit comparé du travail et de la sécurité sociale

Édition imprimée

Date de publication : 1 novembre 2018

Pagination : $92-107$

ISSN : $2117-4350$

Référence électronique

Julie Bourgault et Michel Coutu, «Le système québécois de participation des travailleurs : vers une refondation sur de nouvelles assises constitutionnelles ? ", Revue de droit comparé du travail et de la sécurité sociale [En ligne], 3 | 2018, mis en ligne le 01 novembre 2021, consulté le 13 novembre 2021. URL : http://journals.openedition.org/rdctss/1904; DOI : https://doi.org/10.4000/rdctss. 1904

\section{(c) $(1)$}

Revue de droit comparé du travail et de la sécurité sociale est mise à disposition selon les termes de la Licence Creative Commons Attribution - Pas d'Utilisation Commerciale - Pas de Modification 4.0 International. 


\section{LE SYSTÈME QUÉBÉCOIS}

DE PARTICIPATION DES TRAVAILLEURS :

VERS UNE REFONDATION

SUR DE NOUVELLES ASSISES

CONSTITUTIONNELLES ?

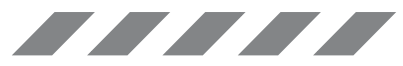

\section{ABSTRACT}

Worker's participation rights, centered around collective bargaining, present numerous weaknesses in Québec. The authors underline in this repect the collision between collective bargaining rights and property rights, including the freedom of commerce. But the new labour constitution in Canada implies, for the authors, revisiting in a positive way basic worker's participation rights

KEYWORDS : Freedom of Association, Collective Bargaining, Labour Constitution, Worker's Participation Rights, Freedom of Commerce and Property Rights.

\section{RÉSUMÉ}

Les auteurs soulignent les faiblesses, en termes de droits de participation, que présente le système actuel de négociation collective au Québec. Les auteurs s'attardent à la collision entre le droit de négociation collective et la liberté d'entreprendre et le droit de propriété. Cependant, la constitutionnalisation du droit du travail en cours au Canada annonce, de l'avis des auteurs, une révision à la hausse des droits de participation des travailleurs.

MOTS CLÉS : Liberté d'association, négociation collective, droits de participation des travailleurs, constitutionnalisation du droit du travail, liberté d'entreprendre et droits de propriété. 


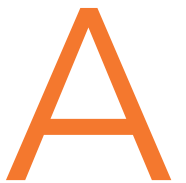

u Québec, le système de participation des travailleurs dans l'entreprise repose essentiellement sur la liberté d'association des travailleurs. En effet, la structuration particulière du droit du travail en contexte québécois demeure fort différente de ce qu'on retrouve en France. Bien que le cadre législatif et conventionnel prévoit des modalités permettant de mettre en $œ u v r e$ la représentation des travailleurs, ces derniers sont confrontés à divers obstacles au plein épanouissement de leurs droits de représentation.

Dans ce qui suit, nous allons d'abord traiter de la structuration particulière des rapports collectifs du travail et du cadre législatif et conventionnel relatif à la représentation des travailleurs (I). Dans un second temps, nous présenterons les obstacles à la participation des travailleurs qui découlent, d'une part, du cadre législatif et, d'autre part, de la prééminence historique des droits de propriété et de la liberté d'entreprendre sur le droit du travail (II). Enfin, nous aborderons sommairement ce que nous nommons la « nouvelle Constitution du travail » dans ses diverses composantes, en évaluant l'impact de la Charte canadienne des droits et libertés sur les droits de représentation des salariés (III).

\section{I - LA REPRÉSENTATION DES TRAVAILLEURS AU QUÉBEC : LE CADRE LÉGISLATIF ET CONVENTIONNEL}

Les rapports collectifs du travail sont conditionnés au Québec par une double distinction, entre salariés/non-salariés d'une part, syndiqués/non syndiqués de l'autre'. Cette double distinction trouve son origine dans le Wagner Act adopté en 1935 aux États-Unis ${ }^{2}$. Ainsi, certains employés considérés comme étant des non-salariés au sens du Code du travail sont généralement dépourvus de tout accès à la syndicalisation, à la négociation collective et au droit de grève. C'est en particulier le cas des cadres agissant comme des représentants de l'employeur, même si leur rôle véritable à cet égard demeure très modeste. Les « entrepreneurs » se trouvant en situation de dépendance économique se voient également exclus du champ des rapports collectifs du travail au Québec 3 . Par ailleurs, les « syndiqués» ne sont pas d'abord et avant tout les adhérents individuels à

1 M. Coutu, L.-Lea Fontaine, G. Marceau et U. Coiquaud, Droit des rapports collectifs de travail, 2e éd., Cowansville (Qué.), Éditions Yvon Blais, tome 1, 2013, p. 8 et s.

2 National Labor Relations Act, 29 U.S.C. §§ 151-169.

3 Au contraire de la situation prévalant au niveau fédéral : cf. Code canadien du travail, L.R.C. (1985), ch. L-2, art. 3. Le droit fédéral canadien ne concerne qu'une minorité de salariés, soit environ $10 \%$ de la main-d'œuvre concentrée toutefois dans des secteurs stratégiques (aéronautique, télécommunications, transport interprovincial, etc.). 
un syndicat, mais les membres d'une unité de négociation (par exemple, les ouvriers de la production dans un établissement manufacturier ou les cols blancs à l'emploi d'une administration municipale) qui est représentée par un syndicat accrédité. Soulignons que le taux de syndicalisation au Québec est d'environ $40 \%$ de la main-d'œuvre, un chiffre relativement stable depuis plusieurs décennies ${ }^{4}$.

Pour comprendre la structuration des rapports collectifs de travail au Québec (et partout au Canada), il est nécessaire d'en revenir aux objectifs visés initialement par le Wagner Act, soit : a) l'atteinte de la paix industrielle, afin de favoriser l'essor du commerce et de l'industrie ; b) l'atteinte de l'égalité dans le pouvoir de négociation des travailleurs par rapport aux employeurs, considérant « the inequality of bargaining power between employees who do not possess full freedom of association $»^{5}$.

Du point de vue de la théorie du droit du travail, le paradigme conditionnant le modèle Wagner est généralement qualifié, par opposition au paradigme volontariste (laissez-faire collectif) britannique ${ }^{6}$, de " pluralisme industriel $»^{7}$. On peut y associer deux principes fondamentaux : $1^{\circ}$ la négociation doit essentiellement prendre place au niveau de l'entreprise si ce n'est de l'établissement, et nullement au niveau de la branche ou du secteur, ${ }^{8}$ ce qui représente pour l'employeur le gage d'une paix industrielle s'étendant au minimum sur quelques années ; $2^{\circ}$ par la voie de la négociation décentralisée se réalise l'équilibre des forces dans l'entreprise, les travailleurs compensant ainsi l'inégalité initiale de pouvoir entre le salarié isolé et l'employeur, et parvenant, entreprise par entreprise, à une forme de démocratie industrielle.

Sur le plan normatif, les principales composantes de la Loi Wagner, livrant l'architecture de base du Code du travail du Québec, peuvent être ainsi décrites:

a) Le syndicat justifiant de l'appui de la majorité des salariés dans une unité de négociation a droit d'obtenir une accréditation formelle de la part de l'État ;

b) Cette accréditation est octroyée par un tribunal administratif réputé neutre et impartial;

c) La loi protège les salariés contre la discrimination antisyndicale, tels le refus d'embauche, le congédiement et autres sanctions pour activités syndicales;

d) Le syndicat accrédité dispose d'un monopole de représentation visant l'ensemble des salariés membres de l'unité de négociation, c'est-à-dire qu'il en est l'unique agent négociateur ;

e) Suite à la transmission d'un avis de négociation, les parties (l'employeur et le syndicat) sont tenues de négocier de bonne foi, en vue de conclure une convention collective ;

4 Cf. A. Labrosse, La présence syndicale au Québec en 2016, Québec, ministère du Travail, de l’Emploi et de la Solidarité sociale, 2017.

5 National Labor Relations Act, précité (extrait du préambule).

6 Sur le volontarisme, voir R. Dukes, The Constitution of Labour. The Enduring Idea of Labour Law, Oxford University Press, 2014, p. 69 et s. Au Canada, J. Fudge, E. Tucker, Labour before the Law. The Regulation of Worker's Collective Action in Canada, 1900-1948, Toronto, University of Toronto Press, 2001, p. 51 et s.

7 Voir K. V. Stone, "The Post-War Paradigm in American Labor Law », Yale Law Journal, 1981, n 90, p. 1509-1580.

8 Celle-ci est en pratique irréalisable, vu l'obligation de paix industrielle découlant de l'existence d'une convention collective en vigueur. 
f) La convention collective ainsi négociée a pleine valeur juridique et lie l'ensemble des salariés membres de l'unité de négociation, qu'ils adhèrent ou non au syndicat accrédité.

Dans le contexte canadien, la réception du Wagner Act s'est effectuée en 1944, dans le contexte particulier du dirigisme économique lié à l'effort de guerre alors en cours. C'est donc essentiellement le gouvernement fédéral qui régulait les relations de travail ; il adopta en 1944 le modèle Wagner, via l'arrêté en conseil n $1003^{9}$, bientôt imité par l'ensemble des provinces dont le Québec ${ }^{10}$, celles-ci retrouvant suite à l'arrêt des hostilités leur compétence exclusive sur l'essentiel des rapports de travail.

Par la suite, il y eut un mouvement graduel, mais fondamental, de «canadianisation » du modèle wagnérien. En effet, les systèmes canadien et américain des relations industrielles ont suivi des voies de plus en plus divergentes. II existe maintenant des différences fondamentales entre ces deux variantes du modèle Wagner, par exemple la garantie, partout au Canada, du maintien de l'emploi en cas de participation à une grève légale, ce qui n'existe pas en droit états-unien ${ }^{11}$. Cette distance juridique explique, en partie du moins, les disparités entre les taux de présence syndicale aux USA, au Canada et, davantage encore, au Québec ${ }^{12}$.

Qui plus est, on peut qualifier de modèle hybride le droit des rapports collectifs du travail en vigueur au Québec. En effet, tout en retenant la grammaire de base du modèle Wagner, celui-ci a été davantage influencé par le droit européen que ce ne fut le cas ailleurs au Canada. En particulier, la négociation de branche, imposée par voie législative, prévaut dans le secteur de la construction ${ }^{13}$ et dans le secteur public au Québec ${ }^{14}$ : il s'agit là d'une situation unique au Canada. En outre, le droit québécois du travail a mis de l'avant certaines solutions originales, telles l'interdiction des travailleurs de remplacement (briseurs de grève), en cas d'exercice légal du droit de grève ${ }^{15}$.

Cependant, le droit québécois du travail ignore en principe le modèle dualiste de représentation (syndicats et comités d'entreprise) qui caractérise la plupart des systèmes européens de relations industrielles. Comme le veut le modèle Wagner, c'estessentiellement par un système fortement décentralisé de négociation collective, avec reconnaissance par accréditation étatique du seul syndicat majoritaire au regard d'une unité de négociation, que se réalise la participation des travailleurs à la gestion des relations de travail dans

9 C.P. 1003, 17 février 1944; Gazette du travail, 1944, vol. 44, p. 146.

10 Loi sur les relations ouvrières, S.R.Q 1941, c. 162A.

11 Outre le congédiement « at will » qui n'existe pas au Canada, mentionnons entre autres l'arbitrage obligatoire des griefs, la possibilité d'un arbitrage obligatoire des différends en cas d'impasse dans la négociation d'une première convention collective, l'imposition par voie législative du précompte syndical, etc. Pour un aperçu, voir M. Coutu, L.L. Fontaine, G. Marceau et U. Coiquaud, Droit des rapports collectifs de travail, op. cit., p. 19 et s.

12 Ce taux est de $38.6 \%$ au Québec, contre $29.2 \%$ au Canada anglais et seulement $12 \%$ aux ÉtatsUnis. (A. Labrosse, La présence syndicale au Québec en 2016, op.cit., p. 4).

13 Loi sur les relations de travail (...) dans l'industrie de la construction, RLRQ c R-20.

14 Loi sur le régime de négociation des conventions collectives dans le secteur public, RLRQ c R-8.2.

15 Code du travail du Québec, art. 109.1. 
l'entreprise ${ }^{16}$. Certes, la législation du travail oblige parfois l'entreprise à se doter d'instances représentatives du personnel, y compris dans le secteur non syndiqué : par exemple, la Loi sur la santé et la sécurité du travail oblige les employeurs à doter l'entreprise de comités paritaires de santé et de sécurité du travail ${ }^{17}$. En vertu de la Loi sur les normes du travail, en cas de licenciements collectifs, des comités paritaires de reclassement des salariés doivent être mis sur pied, etc. ${ }^{18}$ Toutefois, en l'absence d'une représentation syndicale dans l'entreprise, de tels comités semblent dépourvus d'une réelle efficacité.

Par ailleurs, il est rarissime, a fortiori, que des représentants des travailleurs puissent siéger au conseil d'administration des entreprises, même si le Code du travail envisage sommairement cette possibilité. Ainsi, la plus importante société d'État, Hydro-Québec, n'admet aucun délégué des salariés au sein de son conseil d'administration, alors qu'EDF, son équivalent français, fait place à 6 délégués du personnel sur 18 membres du conseil ayant droit de vote ${ }^{19}$.

Soulignons que dans le cas des entreprises syndiquées, les conventions collectives forment des documents volumineux qui couvrent généralement, de manière très détaillée, tous les aspects des rapports de travail, de l'affichage des postes et du recrutement jusqu'à la discrimination au travail, en passant par les congés, heures de travail, l'ancienneté, la formation, etc. ${ }^{20}$. Le respect des dispositions des conventions collectives est assuré par une instance spécialisée, l'arbitre des griefs, lequel est nommé et rémunéré conjointement par les parties patronale et syndicale. Qui plus est, une instance paritaire présente dans plusieurs conventions collectives, souvent désignée "comité de relations du travail », se réunit sur une base régulière pour discuter des questions liées aux relations professionnelles dans l'entreprise ou dans l'institution.

\section{II - LES OBSTACLES AUX DROITS DE REPRÉSENTATION DES TRAVAILLEURS}

Des obstacles majeurs font écran à la pleine représentation des intérêts des travailleurs, découlant d'une part du cadre législatif $(A)$ et d'autre part, de la position prééminente accordée historiquement par les tribunaux aux droits de propriété et à la liberté d'entreprendre (B).

16 Pour un exposé classique, cf. H. Arthurs, "Developing Industrial Citizenship: A Challenge for Canada's Second Century", Canadian Bar Review, 1967, vol. 45. n 4, p. 786-830.

17 Loi sur la santé et la sécurité du travail, RLRQ c. S-2.1, art. 68 et s.

18 Loi sur les normes du travail, RLRQ c. N-1.1, art. 84.0.9.

19 Voir la Loi sur Hydro-Québec, RLRQ c. H-5, art. 4 et s. Cf. http://www.hydroquebec.com/a-propos/ gouvernance/conseil-administration.html

Comp. https://www.edf.fr/groupe-edf/qui-sommes-nous/gouvernance/conseil-d-administration

20 À la différence de la France où l'élément de base est la convention collective de branche complétée par de nombreux avenants et parfois par des accords d'entreprise, au Québec la convention collective conclue au niveau de l'établissement ou de l'entreprise, complétée le cas échéant par des lettres d'ententes, représente le document essentiel. Ce n'est que dans le secteur public québécois où la situation se rapproche le plus de ce qu'on connaît en France : l'entente principale réside dans les conventions collectives (nationales) et les ententes sectorielles, complétées par des ententes régionales et des accords locaux. 


\section{A - LE CADRE LÉGISLATIF}

La présentation du cadre législatif québécois dérivé du modèle Wagner et de son interprétation par les tribunaux spécialisés ou de droit commun permet d'identifier certaines faiblesses caractérisant les droits de représentation des travailleurs au Québec:

1. La définition restrictive de la notion de salarié, laquelle exclut tout employé considéré comme détenteur d'une autorité hiérarchique sur ses collègues, même si celle-ci apparaît minime dans les faits; cette définition n'est pas le fruit du hasard, elle correspond à la vision dualiste - et nullement - pluraliste de l'entreprise, valable à l'échelle de l'Amérique du Nord;

2. La conception résiduaire des droits de direction de l'employeur, laquelle prévaut majoritairement chez les arbitres de griefs et se traduit par une lecture restrictive des dispositions de la convention collective. Celle-ci est vue comme créant autant d'exceptions aux droits de direction de l'employeur, lesquels conservent autrement leur plénitude; une telle perspective apparaît incompatible avec la reconnaissance d'un véritable droit à la codétermination par les travailleurs de la gestion de l'entreprise ${ }^{21}$;

3. La non reconnaissance du syndicalisme minoritaire. Seul le syndicat disposant de l'appui majoritaire des salariés membres d'une unité d'accréditation a le droit de négocier collectivement avec un employeur. Autrement dit, le syndicat accrédité dispose d'un monopole de représentation visant l'ensemble des salariés concernés, tant qu'il bénéficie de leur appui majoritaire. S'insère ici une logique du tout ou rien : si au terme d'une campagne de syndicalisation, le syndicat en gestation n'obtient pas le soutien de la majorité absolue des travailleurs membres de l'unité de négociation projetée, il ne sera pas accrédité par l'État. Même si ce syndicat jouit parmi les salariés d'appuis non négligeables (de l'ordre par exemple de $35 \%$ ou plus), la campagne de syndicalisation sera tout simplement un échec. La minorité favorable à la présence d'un syndicat sera dépourvue de toute possibilité de négociation collective avec l'employeur. Qui plus est, même si en « champ libre » une requête en accréditation peut validement être présentée en tout temps, en pratique, en raison des coûts, du travail acharné et de la mobilisation considérable de ressources qu'implique une démarche de syndicalisation, il faudra attendre longtemps avant de voir, si jamais c'est le cas, le dépôt d'une nouvelle requête en accréditation ;

4. L'obligation législative de paix industrielle en cours de convention collective. Cette obligation législative, laquelle n'existe pas même aux États-Unis, entraîne deux conséquences majeures:

a) d'une part, sauf la présence (fort rare) d'une clause autorisant la renégociation de certains sujets en cours de validité des conventions collectives, les syndicats ne peuvent obliger l'employeur à négocier, durant cette période, même des objets d'importance capitale non prévus à la convention collective par exemple, en cas de structuration de l'entreprise, de licenciements collectifs, de sous-traitance ou de fermeture totale ou partielle ${ }^{22}$. L'employeur est donc libre de déterminer à sa guise

21 La thèse des droits résiduaires, très majoritaire, s'oppose à celle des limitations implicites voulant que " dès qu'une association syndicale a été accréditée et que la négociation collective est engagée dans un établissement », il se produit un changement dans la nature des rapports de travail impliquant que "l'employeur n'a plus seul le droit de tout décider dans son établissement" »: G. Vallée, J. Bourgault, "Cadre juridique de la convention collective », dans P. Jalette, M. Laroche et G. Trudeau, (dir.), La convention collective au Québec, Montréal, Chenelière Éducation, $3^{\mathrm{e}}$ éd., 2017, 21, p. 45-47.

22 Art. 107 C.t. 
l'avenir de l'établissement ou de l'entreprise, sans droit de regard du syndicat, à la condition de respecter les dispositions en vigueur des conventions collectives ${ }^{23}$. L'obligation de paix industrielle a produit des effets négatifs sur la représentation des intérêts des salariés, en particulier dans les cas les plus décisifs, ceux qui font appel à de profondes restructurations. Or, de telles restructurations se sont faites de plus en plus fréquentes, sous la pression de la mondialisation ${ }^{24}$; elles n'obéissent pas uniquement à des logiques économiques, mais sont fréquemment utilisées pour réduire l'influence syndicale au niveau de l'entreprise ${ }^{25}$. Le mouvement syndical a été pris de court par le rythme des restructurations en particulier dans le secteur privé, et fréquemment n'a pas été en mesure de le contrer de manière efficace ;

b) d'autre part, l'impossibilité pour les travailleurs de faire la grève en cours de négociation collective, pour quelque motif que ce soit, ce qui inscrit un déséquilibre structurel dans les rapports de travail, au seul bénéfice des employeurs. Les sanctions des grèves "illégales ", pouvant aller jusqu'au congédiement des salariés et impliquant de fortes amendes pour les organisations syndicales, se sont généralement révélées suffisamment dissuasives pour empêcher les syndicats de recourir à l'action économique en cours de convention collective.

\section{B - LES DROITS DE PROPRIÉTÉ ET LA LIBERTÉ D'ENTREPRENDRE}

Au regard de la «constitution économique » du type nord-américain ${ }^{26}$, la syndicalisation, la négociation collective et l'exercice de moyens de pression (tels la grève et le piquetage) sont généralement perçus comme érigeant autant d'obstacles à la profitabilité optimale des entreprises. Certes, historiquement, nombre d'employeurs, en particulier les grandes entreprises, ont trouvé somme toute avantageux de s'adonner avec les organisations syndicales à une gestion commune, à l'externe, du marché du travail et, à l'interne, des rapports de travail dans l'entreprise. Mais si on considère en particulier l'exemple américain, cette époque apparaît révolue pour nombre de firmes importantes aux États-Unis lesquelles cherchent à éviter la syndicalisation, par exemple en délocalisant leurs activités dans les États du sud, plus réfractaires à la présence des syndicats. Dans le commerce de détail, entre autres, la syndicalisation est considérée comme incompatible avec le modèle d'affaire dominant le capitalisme concurrentiel, lequel nécessite une rémunération de la main-d'œuvre au plus bas coût possible.

23 Pour une critique de cette situation, voir P. Verge, Le droit de grève. Fondements et limites, Montréal, Éditons Yvon Blais, 1985, p.149 et s; G. Trudeau, "La grève au Canada et aux États-Unis: d'un passé glorieux à un avenir incertain », Revue juridique Thémis, 2004, n³8, p. 1-48, p. 42 et s.

24 Cf. M. Coutu, J. Bourgault, « Le droit du travail et les restructurations industrielles au Canada : une pluralité normative », dans C. Didry, A. Jobert (dir.), L'entreprise en restructuration. Dynamiques industrielles et mobilisations collectives, Rennes, Presses universitaires de Rennes, 2010, p. 107-120.

$25 \mathrm{~V}$. ci-dessous, la section suivante consacrée aux fermetures d'entreprises motivées par l'antisyndicalisme.

26 Sur la notion de " constitution économique », voir notamment, G. Teubner, "Transnationale Wirtschaftsverfassung: Franz Böhm und Hugo Sinzheimer jenseits des Nationalstaates ", Zeitschrift für ausländisches öffentliches Recht und Völkerrecht, 2014, 74, p. 733-761. 
L'implantation au Canada d'une multinationale américaine antisyndicale comme l'est Wal-Mart n'a pas manqué, par ricochet, d'inciter d'autres employeurs à se montrer hostiles à la syndicalisation ${ }^{27}$, en invoquant au besoin le droit de propriété et la liberté d'entreprendre pour fermer des établissements, à seule fin de prévenir l'implantation d'un syndicat. D'ailleurs, dans les dernières années, plusieurs cas de fermeture d'établissement au Québec, survenant au stade de l'accréditation ou de la négociation de la convention collective, ont donné lieu à de nombreuses décisions judiciaires ${ }^{28}$.

À travers l'étude de la jurisprudence portant sur la fermeture d'entreprise motivée par l'antisyndicalisme, nous constatons la place centrale accordée historiquement par les tribunaux à la liberté d'entreprendre et au droit absolu de " cesser de faire affaire " (en dépit du flou de l'expression) ${ }^{29}$. Un bref rappel de l'affaire Wal-Mart nous paraît essentiel pour bien situer la question. En Amérique du Nord, aucun établissement de cette firme multinationale n'était syndiqué avant que le Wal-Mart de Jonquière ${ }^{30}$ ne fasse l'objet d'une accréditation syndicale octroyée en août 2004 aux Travailleurs unis de l'alimentation et du commerce (TUAC) par la Commission des relations du travail (CRT ci-après) ${ }^{31}$. Les négociations traînant en longueur, le syndicat requit la nomination d'un arbitre de différends en vue de la conclusion d'une première convention collective, tel que le prévoit le Code du travail $^{32}$. Or, le jour même où le ministre du Travail du Québec nommait un arbitre, Wal-Mart annonça la fermeture prochaine de son établissement de Jonquière, prétendument pour raisons économiques.

27 V. M.Coutu, "Licenciements collectifs et fermetures d'entreprise au Québec: Le cas Wal-Mart », Travail et emploi, 2007, n 109, pp. 39-50.

28 Relevons 20 décisions chez Wal-Mart [dont 18 pour le seul magasin de Jonquière], 7 décisions chez Zellers (magasins à grande surface) et 7 décisions au stade interlocutoire uniquement chez Couche-Tard (alimentation de proximité).

Depuis, d'autres litiges du même type sont apparus, notamment dans le commerce de détail: cf. Travailleurs et travailleuses unis de l'alimentation et du commerce, section locale 501 et Groupe BMTC inc., 2017 QCTAT 892 (ameublement et appareils électroniques). V. aussi Syndicat des salariés des services d'alarme (CSD) (Section ADT) et Services de sécurité ADT Canada inc., 2018 QCTAT 1000 (fermeture motivée par l'anti-syndicalisme d'un centre d'assistance technique). Travailleurs et travailleuses unis de l'alimentation et du commerce, section locale 501 et Ville de Rosemère, 2017 QCTAT 5606 (fermeture complète d'un service d'incendie municipal); Travailleurs et travailleuses unis de l'alimentation et du commerce, section locale 501 c Rosemère (Ville), 2017 CanLII 71832 (CC SAT) - (même situation, examinée toutefois sous l'article 59 C.tr.). Voir également : Unifor, section locale 1209 et Delastek inc., 2018 QCTAT 1136 (licenciement collectif de la moitié des salariés en grève légale).

Voir par contre : Syndicat des Métallos, section locale 7016 c. Systèmes et câbles d'alimentation Prysmian Canada ltée, 2011 QCCRT 116 (fermeture non sanctionnée d'un établissement suite au rejet des offres patronales par les travailleurs syndiqués).

29 En ce sens que la jurisprudence confond fréquemment l'entreprise et l'établissement, alors qu'il s'agit de notions juridiques fort différentes.

30 Jonquière (60 000 heures) est l'un des trois arrondissements formant la ville de Saguenay au Québec.

31 Devenue en 2016 le Tribunal administratif du travail (TAT).

32 L'article 93.1. du Code du travail du Québec prévoit qu'en cas d'impasse, une 1 ère convention collective peut être imposée par un arbitre de différends nommé par le ministre du Travail, de manière à assurer le parachèvement du processus de syndicalisation. 
Le principal recours intenté par les TUAC se fondait sur l'article 15 du Code du travail qui protège l'emploi du salarié exerçant des activités syndicales, avec droit à la réintégration avec pleine indemnisation en cas de congédiement ${ }^{33}$. En un tel cas, l'article 17 du Code du travail crée une présomption en faveur du salarié, facilitant la preuve d'un comportement illicite de la part de l'employeur. En effet, lorsque le salarié démontre l'exercice d'activités syndicales de façon concomitante à la sanction, celle-ci est présumée avoir été prise en raison d'un animus antisyndical. L'employeur doit alors impérativement démontrer, par prépondérance de preuve, la présence d'une autre cause « juste et suffisante » justifiant l'imposition de la sanction.

Toutefois, la Cour suprême du Canada (CSC ci-après) décida à la majorité, dans un arrêt de 2009, que ce recours ne pouvait être utilisé en cas de fermeture d'entreprise ou d'établissement lorsque la fermeture est "réelle, définitive et authentique ", quels qu'en soient les motifs ${ }^{34}$. De manière discutable ${ }^{35}$, la Cour mettait en effet en exergue, invoquant des décisions de l'ancien Tribunal du travail datant des années 80, le « droit de l'employeur de cesser de faire affaire, et ce, même pour des motifs condamnables socialement $»^{36}$. En d'autres mots, dans le cadre d'un recours fondé sur l'article 15 du Code du travail, le décideur ne devrait pas s'arrêter aux motifs de la fermeture : au contraire, le simple constat d'une fermeture réelle et définitive serait suffisant pour justifier les licenciements. En l'espèce, la preuve démontrait que tel était le cas : selon la Cour, le lieu de travail n'était plus en activité ${ }^{37}$.

Par conséquent, le droit de l'employeur de cesser de faire affaire, même pour des motifs socialement condamnables (un droit qui n'est par ailleurs pas du tout affirmé explicitement par la loi au Québec), neutralisait complètement la protection législative de la liberté syndicale, en l'occurrence les congédiements liés à l'exercice du droit de syndicalisation. Cependant, de notre perspective, le récent mouvement de constitutionnalisation du droit du travail au Canada permet d'endiguer ce courant et d'affirmer au contraire, dans une mesure qui reste à être précisée, la prépondérance de la liberté syndicale face aux droits de propriété et à la liberté d'entreprendre.

33 Cette disposition ne protège pas seulement les responsables syndicaux, mais tout salarié contre toute forme de discrimination antisyndicale.

34 Plourde c. Compagnie Wal-Mart du Canada Inc., [2009] 3 RCS 465.

35 Voir les motifs dissidents des juges Lebel, Abella et Cromwell (ibid, par. 66 et s.), de même que la réaction généralement négative de la doctrine.

36 City BuickPontiac (Montréal) Inc. c. Roy, [1981] T.T. 22, suivi par: Caya c. 16419749 Québec Inc., D.T.E. 85T-242 (T.T); Bérubé c. Groupe Samson Inc., D.T.E. 85T932 (T.T.); Ouellette c. Restaurants Scott Québec Ltée, D.T.E. 88T546, (T.T.); Entreprises Bérou inc. c. Arsenault, [1991] T.T. 312; Plourde c. Compagnie Wal-Mart du Canada Inc., 2009 CSC 54, [2009] 3 R.C.S. 465.

37 Incidemment, la Cour rejetait du revers de la main toute pertinence de la liberté constitutionnelle d'association en ce domaine, estimant que celle-ci ne régit pas les rapports de droit privé. Ce faisant, la CSC refusait implicitement de prendre en considération la Charte des droits et libertés de la personne du Québec, laquelle est pourtant applicable à des rapports de cette nature et garantit par ailleurs la liberté d'association en son article 3. 


\section{III - LA CONSTITUTIONNALISATION DU DROIT DU TRAVAIL : IMPACT SUR LES DROITS DE PARTICIPATION DES TRAVAILLEURS}

Depuis le revirement jurisprudentiel de 2007 de la CSC relatif à la portée de l'article 2d) de la Charte canadienne des droits et libertés, le droit canadien des rapports collectifs de travail connaît un important mouvement de constitutionnalisation. En effet, renversant sa jurisprudence antérieure, très formaliste et imprégnée de libéralisme économique ${ }^{38}$, la Cour a consacré le droit de négociation collective, puis le droit de grève, en tant que droits constitutionnels fondamentaux, ce qu'elle s'était totalement refusée à faire antérieurement. À notre avis, ce revirement jurisprudentiel revêt une importance considérable, dont la portée exacte demeure toutefois à être précisément définie. La constitutionnalisation en cours institue vraisemblablement une troisième étape historique dans l'évolution du droit collectif du travail au Canada, après l'ère du volontarisme ou « laissez-faire collectif » du type britannique (qui va approximativement de 1872 à 1944), puis celle de l'institutionnalisme (ou " pluralisme industriel ») engendré par la réception législative du modèle Wagner (1944-2007). Elle a un impact à la fois sur la représentation des travailleurs liée au cadre législatif $(A)$ et sur l'interprétation par les tribunaux du sens des droits de propriété et de la liberté d'entreprendre (B).

\section{A - LE CADRE LÉGISLATIF}

Afin d'illustrer l'impact de la constitutionnalisation du droit du travail, nous pouvons d'abord reprendre brièvement les quatre points développés ci-dessus (section I) mettant en exergue autant de faiblesses relatives aux droits de représentation des salariés au Canada/Québec :

1. L'accès à la syndicalisation. Depuis le tournant de la CSC en faveur de la constitutionnalisation du droit du travail, plusieurs groupes de travailleurs, autrefois dépourvus de tout droit à la représentation collective par diverses dispositions législatives, se sont vus reconnaître par les tribunaux le droit à la syndicalisation. Mentionnons par exemple les membres de la Gendarmerie royale du Canada, les travailleurs agricoles migrants et les "ressources intermédiaires " dans le secteur de la santé et des services sociau ${ }^{39}$. Autre développement très important, en décembre 2016, le TAT a jugé contraire à la liberté constitutionnelle d'association l'exclusion des cadres de l'accès à la syndicalisation au Québec. La décision du TAT fait l'objet d'un processus de révision judiciaire qui s'annonce fort long, l'État entendant défendre cette exclusion, laquelle concerne potentiellement

38 V. la «trilogie » de 1987: Renvoi relatif à la Public Service Employee Relations Act (Alb.), [1987] 1 R.C.S. 313 (ci-après Renvoi) ; SDGMR c. Saskatchewan, [1987] 1 R.C.S. 460 et AFPC c. Canada, [1987] 1 R.C.S. 424.

39 Voir : Association de la police montée de l'Ontario c. Canada (Procureur général), 2015 CSC 1, [2015] 1 R.C.S. 3 (policiers fédéraux). Quant aux travailleurs agricoles, cf. Dunmore c. Ontario (Procureur général), [2001] 3 R.C.S. 1016 ; Ontario (Procureur général) c. Fraser, 2011 CSC 20 ; au Québec : Travailleurs et travailleuses unis de l'alimentation et du commerce, section locale 501 c. L'Écuyer, 2010 QCCRT 191. Voir cependant le régime particulier institué par le gouvernement du Québec concernant les travailleurs agricoles, art. 111.27 à 111.32 C.t. Quant aux « ressources intermédiaires » et autres salariées fournissant à leur domicile divers services en matière de santé et de services sociaux, dont l'État du Québec refusait la syndicalisation, voir Confédération des syndicats nationaux c. Québec (Procureur général), 2008 QCCS 5076. 
des centaines de milliers de cadres au Québec et contredit la vision dualiste, fortement hiérarchisée, de l'entreprise prévalant en Amérique du Nord ${ }^{40}$.

2. La conception résiduelle des droits de direction. Comme nous l'avons mentionné, l'approche traditionnelle en droit du travail au Canada veut que l'employeur, sous réserve des dispositions expresses de la convention collective, conserve la plénitude de ses droits résiduels de direction et puisse agir ainsi à sa guise ${ }^{41}$. Dans une décision récente, la CSC précise toutefois que l'employeur, au-delà de la bonne foi, doit alors prendre des décisions qui soient objectivement raisonnables et équitables ${ }^{42}$. À cette fin, poursuit la Cour, il doit être procédé à une évaluation axée sur la « mise en balance des intérêts » des salariés et de l'employeur : en particulier, l'arbitre des griefs doit d'une part, analyser le cadre de travail tel que le définit la convention collective et, d'autre part, déterminer l'objectif recherché par l'employeur et évaluer le moyen choisi par celui-ci pour l'atteindre ${ }^{43}$. Bien que la CSC ne s'appuie pas à cet égard sur des normes constitutionnelles, cette approche objective axée sur la mise en balance des intérêts quant à l'exercice des droits résiduels de l'employeur est certainement plus compatible avec celles-ci que la simple exigence de la bonne foi. II suffit de faire référence ici à l'opinion des juges Mc. Lachlin et Lebel dans l'arrêt B.C. Health Services : "la négociation collective permet aux travailleurs de parvenir à une forme de démocratie et de veiller à la primauté du droit en milieu de travail. Ils acquièrent voix au chapitre pour l'établissement des règles qui régissent un aspect majeur de leur vie» ${ }^{44}$.

3. L'impossibilité du syndicalisme minoritaire. Àce jour, les tribunaux n'ont pas été saisis directement de cette question. On relèvera tout au plus un obiter des juges Mc Lachlin et Lebel, quelque peu sibyllin, portant sur le sujet ${ }^{45}$. La doctrine a toutefois fait référence aux positions du BIT, en particulier à celles du Comité de la liberté syndicale (CLS), lesquelles estiment contraire aux principes de la liberté d'association qu'un syndicat minoritaire soit, pour ce seul motif, privé de tout droit de représentation ${ }^{46}$. Rappelons que la CSC considère les décisions du CLS comme représentant une source hautement persuasive d'interprétation de l'article $2 \mathrm{~d}$ ) de la Charte canadienne des droits et libertés ${ }^{47}$. II faut

40 Cf. Association des cadres de la Société des casinos du Québec et Société des casinos du Québec inc., 2016 QCTAT 6870, (Demande de pourvoi en contrôle judiciaire, CS-500-17-097007-176) ; Association professionnelle des cadres de premier niveau d'Hydro-Québec (APCPNHQ) et HydroQuébec, 2016 QCTAT 6871 (Demande de pourvoi en contrôle judiciaire, CS-500-17-097044-179).

41 Tout au plus, suivant le courant majoritaire prévalant chez les arbitres de griefs, l'employeur est-il alors assujetti à un critère de bonne foi, c'est-à-dire qu'il ne doit pas procéder de manière purement arbitraire ou malveillante

42 Association des juristes de justice c. Canada (Procureur général), 2017 CSC 55.

43 Ibid, par. 25.

44 Health Services and Support - Facilities Subsector Bargaining Assn. c. Colombie-Britannique, [2007] 2 RCS 391, 2007 CSC 27, par. 85.

45 «L'alinéa 2d) n'impose cependant pas un processus où chaque association finira par obtenir la reconnaissance qu'elle cherche (...) Comme nous l'avons dit, il peut aussi être satisfait à l'al. 2d) en présence d'un modèle reposant sur la majorité et l'exclusivité (tel que le modèle fondé sur la loi Wagner) qui impose des restrictions aux droits des individus afin de réaliser des objectifs communs »: Association de la police montée de l'Ontario c. Canada (Procureur général), 2015 CSC 1, [2015] 1 R.C.S., par.98.

46 Voir en ce sens R. J. Adams, "Bringing Canadass Wagner Act Regime into Compliance with International Human Rights Law and the Charter ", Canadian Lab. \& Emp. L.J., 2015-2016, n 19, p. 365-398.

47 Saskatchewan Federation of Labour c. Saskatchewan, [2015] 1 RCS 245, 2015 CSC 4, par. 69. 
accorder une importance décisive au fait que la CSC s'est refusée à constitutionnaliser le modèle Wagner, fondé sur la seule représentation majoritaire, aux fins de la délimitation des bases normatives de la liberté syndicale au Canada : la Cour a souligné à maintes reprises que des modèles alternatifs de représentation syndicale peuvent tout aussi bien servir les fins de la négociation collective. Considérant en outre les obligations internationales qui incombent au Canada en vertu des conventions $n^{\circ} 87$ et $n^{\circ} 98$ de l'OIT ${ }^{48}$, il nous apparaît que lorsqu'un syndicat dispose d'un appui significatif au sein d'un groupe de salariés, sans parvenir toutefois à obtenir un appui majoritaire comme l'exige le modèle Wagner, ce syndicat devrait avoir accès à un processus de négociation collective de bonne foi auprès de l'employeur concerné, du moins en ce qui concerne ses adhérents. La reconnaissance de cette possibilité est impérative à notre avis pour mettre en conformité la législation canadienne et québécoise du travail avec le droit international. Cette possibilité permettrait à l'évidence à une part substantielle des salariés non-syndiqués de parvenir à une forme de négociation collective avec leurs employeurs ${ }^{49}$.

4. L'obligation de paix industrielle. La validité de l'interdiction législative de toute grève lorsqu'une convention collective est en vigueur, indépendamment de la question en litige, a récemment été mise en doute devant certains tribunaux d'arbitrage, à ce jour sans succès ${ }^{50}$. Cette interdiction, caractéristique de la législation du travail partout au Canada, s'étend également, nous l'avons vu, aux sujets non couverts par les conventions collectives, le cas échéant en cas de restructuration de l'entreprise décidée unilatéralement par l'employeur, en dehors des périodes légales de négociation collective. L'illégalité de tout moyen de pression même lorsque l'emploi des travailleurs est en jeu explique en partie l'impuissance des syndicats du secteur privé à faire face aux licenciements collectifs massifs survenus dans plusieurs secteurs industriels au Québec, tels la foresterie ou la métallurgie. À notre avis, cette interdiction généralisée des grèves imposée par la Loi n'est pas compatible avec la reconnaissance, depuis 2015, du droit de grève comme droit constitutionnel fondamental des travailleurs ${ }^{51}$.

\section{B - L'IMPACT SUR LE DROIT DE PROPRIÉTÉ ET DE LA LIBERTÉ D'ENTREPRENDRE}

Bien que, dans l'état actuel du droit, la liberté d'entreprendre et le droit de cesser de faire affaire occupent toujours une place importante, ils n'immunisent plus l'employeur contre toute sanction lors d'une fermeture antisyndicale. Même si des flottements demeurent dans la jurisprudence, le mouvement récent de constitutionnalisation du droit du travail semble inciter les tribunaux administratifs du travail et les tribunaux de droit commun à mettre

48 D'autant que le Canada vient de ratifier la convention n 98 (juin 2017).

49 Le principe du syndicalisme minoritaire s'appliquerait donc au seul secteur actuellement nonsyndiqué. Quant au secteur syndiqué, compte tenu du modèle Wagner qui demeure légitime dans ses fondements, celui-ci demeurerait régi par le principe majoritaire. Autrement dit, un syndicat passant d'un appui minoritaire substantiel à un appui majoritaire des salariés concernés bénéficierait dès lors du monopole de la représentation, avec toutes les conséquences qui en découlent. Une telle situation nous apparaît conforme aux exigences du droit international du travail.

50 V. par exemple Corporation d'urgences-santé c Syndicat du préhospitalier FSSS-CSN, 2017 CanLII 81906 (OC SAT).

51 Saskatchewan Federation of Labour c. Saskatchewan, décision précitée. 
bien davantage au premier plan la liberté d'association, plutôt que d'affirmer la primauté du droit de propriété de l'employeur et de sa liberté d'entreprendre. Examinons certains litiges récents relatifs à l'entrave antisyndicale (1) et au maintien des conditions de travail (2).

1. Paradoxalement, la décision formaliste et restrictive de la CSC dans l'affaire WalMart (2009) contenait elle-même en germes les éléments d'une évolution ultérieure de la jurisprudence beaucoup plus respectueuse du droit à la syndicalisation. En particulier, si les articles 15 et suivants du Code du travail ne sont d'aucune utilité aux salariés licenciés du fait de l'interprétation littérale du texte qui prévaut toujours, la Cour ouvrait la porte, en obiter dictum, au large champ d'application de la notion " d'entrave antisyndicale » (art. 12 à 14, Code du travail), même en cas de fermeture définitive d'un établissement ou d'une entreprise ${ }^{52}$. Le cas échéant, il sera possible d'évaluer les motifs mêmes de la fermeture puisque celle-ci ne fait pas écran, même si réelle et définitive, à la mise en œuvre de plaintes d'entrave antisyndicale, contrairement aux articles 15 et s. du Code du travail ${ }^{53}$.

Ainsi, plusieurs plaintes fondées sur la notion d'entrave furent déposées concernant la fermeture de certains « dépanneurs ${ }^{54}$ Couche-Tard à Montréal survenue en plein processus de négociation collective. Dans une décision interlocutoire, la CRT a refusé d'accorder une ordonnance provisoire de maintien des opérations, en se basant sur le droit de cesser de faire affaire ${ }^{55}$. Elle a toutefois souligné que s'il s'agissait réellement de fermetures antisyndicales, le tribunal pourrait, lors de l'audition au fond, ordonner la réintégration des salariés, avec indemnité compensatoire, dans d'autres établissements ${ }^{56}$. Une convention collective a finalement été conclue et le syndicat s'est désisté des plaintes.

Puisque, contrairement à l'article 17 C.t., aucune présomption légale d'animus antisyndical ne peut être invoquée au regard d'allégations d'entrave ou d'ingérence, le syndicat devra démontrer que la fermeture de l'entreprise était fondée sur un tel motif. A priori, le fardeau de preuve incombant au syndicat semble lourd selon la jurisprudence traditionnelle ${ }^{57}$. Cependant, dans la décision Plourde c. Wal-Mart de 2009, la CSC soulignait que la preuve d'un animus antisyndical en cas d'entrave au sens du Code du travail n'obéit pas au critère de la preuve déterminante : la présence d'un seul considérant antisyndical suffit à démontrer l'illégalité du comportement de l'employeur, même si d'autres motifs valides, d'ordre économique par exemple, fondent la décision de fermeture. En d'autres termes, le critère de la causalité adéquate suffit à établir le bien-fondé de la plainte, soit l'existence d'une possibilité objective de lien causal entre un motif illicite dont la présence est établie et la décision de cesser de faire affaire, même si le syndicat n'est pas en mesure d'en démontrer la nature déterminante. Une telle approche est bien évidemment apte à

52 Id. Voir aussi Travailleurs et travailleuses unis de l'alimentation et du commerce, section locale 503 c. Compagnie Wal-Mart du Canada, 2014 CSC 45 (Wal-Mart 2).

53 ld., par. 64.

54 N.b. au Québec, petit commerce d'alimentation.

55 Syndicat des travailleuses et travailleurs des Couche-Tard de Montréal et Laval - CSN et Couche-Tard inc., 2011 QCCRT 0449. Voir au même effet: Syndicat international des travailleuses et travailleurs de la boulangerie, confiserie, tabac et meunerie, section locale 55 (SITTBCTM) et Lantic inc., D.T.E. 2013 T-68 (T.A.).

562011 QCCRT 449.

57 Voir par exemple Syndicat des Métallos, sections locales 7016 et 8428 c. Systèmes et câbles d'alimentation Prysmian Canada ltée, 2011 QCCRT 0116; Syndicat des travailleurs et travailleuses Au Roi du coq rôti c. Au Roi du coq rôti inc., 2010 QCCRT 0365. 
faciliter de beaucoup la preuve d'un comportement antisyndical en cas d'entrave au sens de l'article 12 du Code du travail, nonobstant l'absence du mécanisme de la présomption légale applicable lors d'un congédiement, suspension, déplacement etc. fondé sur l'exercice d'un droit prévu par le Code (art. 15 et s., Code du travail) ${ }^{58}$.

2. En opérant implicitement un important revirement jurisprudentiel en 2014, la CSC a donné ouverture à un autre type de recours, d'une vaste portée, dans les cas de restructurations, y compris les fermetures antisyndicales. Cette décision ${ }^{59}$, bien qu'elle n'y fasse pas directement référence, s'inscrit à l'évidence dans le mouvement de constitutionnalisation du droit du travail en donnant une interprétation substantielle à une disposition du Code du travail relative au maintien des conditions de travail, autrefois vue comme ayant uniquement une portée procédurale.

Ainsi, dans l'affaire du Wal-Mart de Jonquière, le syndicat avait déposé une autre plainte, devant l'arbitre de griefs cette fois, alléguant que la fermeture de l'établissement constituait une violation de l'article 59 du Code du travail, lequel oblige l'employeur à maintenir les conditions de travail en vigueur, suite au dépôt d'une requête en accréditation ${ }^{60}$. Dans son arrêt de 2014, la Cour suprême a jugé qu'en ce cas ${ }^{61}$, « l'employeur n'est (1) ni protégé par la fermeture de son établissement (2) ni, le cas échéant, libéré de l'obligation de prouver que sa décision est conforme à ses pratiques habituelles de gestion " ${ }^{62}$ : à défaut de respecter l'article 59 du Code du travail, l'employeur devra en assumer les conséquences, y compris en cas de fermeture illégale, notamment en indemnisant les travailleurs concernés pour l'ensemble des préjudices subis. La preuve d'une telle modification des conditions de travail revient certes au syndicat ${ }^{63}$, mais il ne lui est nullement nécessaire, vu la teneur de l'article 59 du Code du travail, de démontrer que la décision « est motivée par un quelconque animus antisyndical $»^{64}$.

58 Voir aussi, au niveau fédéral : Fedex Freight Canada Corp. v. Teamsters Local Union No. 31, 2017 FCA 78 (fermeture d'un centre de services motivé par l'anti-syndicalisme).

59 Travailleurs et travailleuses unis de l'alimentation et du commerce, section locale 503 c. Compagnie Wal-Mart du Canada, [2014] 2 RCS 323 (Ci-après Wal-Mart 2).

60 Cet article prévoit l'interdiction pour l'employeur de modifier les conditions de travail des salariés sans le consentement écrit d'une association de salariés à compter du dépôt d'une requête en accréditation ou de l'expiration de la convention collective et ce, jusqu'à ce que le droit de grève ou de lock-out soit exercé ou qu'une sentence arbitrale soit rendue.

61 Avant cette décision, la jurisprudence considérait que l'exercice des droits de direction de l'employeur de façon habituelle « inclut le droit de cesser ses activités en tout ou en partie »: Alliance internationale des employés de scène et de théâtre, section locale 56 c. Société du Centre PierrePéladeau, [2004] R.J.D.T. 1246. (Requête pour ordonnance de sauvegarde, décision intérimaire en attendant décision sur le fond : Société du Centre Pierre-Péladeau c. Alliance internationale des employés de scène et de théâtre, du cinéma, métiers connexes et des artistes des États-Unis et du Canada (IATSE), section locale 56, [2006] R.J.D.T. 293 (T.A.).

62 Wal-Mart 2, par. 64.

63 ld., par. 46.

64 Id., par. 38 ; Union des routiers, brasseries, liqueurs douces \& ouvriers de diverses industries c. Syndicat des employé-es de SPC Automation (CSN), [1994] T.A. 718; Centre de la petite enfance Casse-noisette inc. c. Syndicat des travailleuses (eurs) en garderie de Montréal, [2000] R.J.D.T. 1859 (T.A.). 
Dès que le syndicat satisfait à ce fardeau de preuve, l'employeur doit alors démontrer que le comportement adopté relève d'une pratique usuelle de gestion ${ }^{65}$ et qu'il n'a procédé qu'aux changements normaux et essentiels à l'administration quotidienne de l'entreprise ${ }^{66}$. En particulier, une modification des conditions de travail devra être cohérente avec les pratiques antérieures de gestion de l'entreprise ou à défaut, être « conforme à la décision qu'aurait prise un employeur raisonnable placé dans les mêmes circonstances $»^{67}$. Ainsi, dans le cadre d'une fermeture d'établissement, l'employeur devra démontrer que des contraintes économiques majeures imposaient raisonnablement une telle décision. WalMart n'étant aucunement en mesure de fournir une preuve convaincante de telles difficultés financières concernant sa succursale de Jonquière - bien au contraire, les faits mis en preuve attestaient de la rentabilité économique de l'établissement -, son geste fut jugé contraire à l'art. 59 du Code du travail. En conséquence, près de dix ans après la fermeture du magasin de Jonquière, Wal-Mart dut finalement indemniser les salariés licenciés, à hauteur de plusieurs millions de dollars ${ }^{68}$.

Soulignons que dans cette décision très importante, la CSC précise l'objet de l'article 59 du Code du travail : celui-ci ne vise pas au seul maintien de «l'équilibre » entre la partie patronale et syndicale ${ }^{69}$, mais cherche avant tout à favoriser la liberté d'association et la négociation collective ${ }^{70}$. Le lien avec le nouveau droit constitutionnel du travail paraît ici évident.

\section{Conclusion}

Dans le contexte canadien, si l'on cherche à répondre à la question «Quels droits de représentation et de participation sont reconnus aux travailleurs? », il y aurait peu à dire, si l'on ne part pas du principe que la négociation collective se déroulant au niveau de l'unité d'accréditation (généralement l'établissement ou l'entreprise) constitue au minimum un équivalent fonctionnel au système des comités d'entreprise présent, avec de multiples variantes, au niveau européen. En outre, il est très rare au Canada que des représentants des salariés siègent aux conseils d'administration des entreprises privées ou publiques: toutefois, en milieu syndiqué, la forte présence syndicale y compris au niveau local permet peu ou prou d'atteindre des résultats similaires en termes d'information ou de consultation.

Certes, nous relevons les nombreuses faiblesses, du point de vue des droits de participation des travailleurs, que présente le système actuel de négociation collective au Canada, à commencer par l'absence d'accès à la syndicalisation pour des catégories importantes d'employés, telles au Québec les cadres et les entrepreneurs économiquement dépendants. De même, l'obligation législative de paix industrielle et le principe de la majorité absolue liée historiquement à la réception du modèle Wagner états-unien représentent autant d'obstacles au plein épanouissement de la négociation collective.

65 Wal-Mart 2, par. 81

66 Travailleuses et travailleurs de l'alimentation et du commerce, section locale 501 c. Wal-Mart Canada (St-Hyacinthe) (grief syndical), D.T.E. $2010 T-797$ (T.A.).

67 Wal-Mart 2, par. 56.

68 Suite à une entente entre les TUAC et Wal-Mart, les termes exacts de l'accord relatif aux indemnités de licenciement demeurent confidentiels.

69 Une perspective que nous rattachons au paradigme du pluralisme industriel, aujourd'hui désuet.

70 V. en ce sens R. v. Canadian Union of Public Employees, Local 1252, 2016 CanLII 67993 (NB LEB), par. 24. 
Nous avons prêté une attention particulière à la collision entre la négociation collective et la liberté d'entreprendre et le droit de propriété, normes fondamentales (encore que non-écrites) de la constitution économique au Canada. La fermeture antisyndicale du magasin Wal-Mart de Jonquière au Québec a semblé consacrer de prime abord la primauté absolue de ces normes économiques sur les droits collectifs des travailleurs. Toutefois, au terme d'un combat épique devant les tribunaux durant une décennie, les 190 salariés du Wal-Mart de Jonquière ont finalement eu gain de cause, touchant des indemnités compensatoires substantielles. Cette victoire en bout de piste devant la CSC serait peu compréhensible sans l'arrière-plan du revirement jurisprudentiel opéré par la Cour, à partir de 2007, en matière de liberté syndicale : un mouvement de constitutionnalisation du droit du travail a été enclenché à partir de ce moment, dont les contours précis demeurent largement indéfinis, mais qui, se réclamant d'emblée d'une pleine conformité aux normes internationales du travail, contient en germe à tout le moins la possibilité d'une transformation en profondeur du système canadien des relations industrielles et des droits de représentation et de participation des travailleurs qui en découlent.

\section{JULIE BOURGAULT}

Professeure agrégée de droit du travail au Département de relations industrielles de l'Université du Québec en Outaouais, membre du Centre de recherche interuniversitaire sur la mondialisation et le travail (CRIMT) et de l'Équipe de recherche travail et société (ERTS).

Thèmes de recherche : Droits et libertés de la personne, liberté d'entreprendre, restructurations d'entreprises, les droits fondamentaux, constitutionnalisation des libertés syndicales, harcèlement psychologique au travail.

\section{Publications :}

Liberté d'entreprendre, Liberté d'association et restructurations d'entreprises, ed. Wilson \& Lafleur, 2016.

" Le régime québécois de protection contre le harcèlement psychologique au travail : particularités et enjeux jurisprudentiels ", Revue de droit comparé du travail et de la sécurité sociale, 2018-2, p. 22.

\section{MICHEL COUTU}

Professeur titulaire de droit du travail et de sociologie du droit à l'École de relations industrielles de I'Université de Montréal, membre du Centre de recherche interuniversitaire sur la mondialisation et le travail (CRIMT).

Thèmes de recherche : Rapports entre droit et économie, transformations de la négociation collective dans la grande entreprise et dans le secteur public au Québec.

\section{Publications :}

Max Weber's Interpretive Sociology of Law, Routledge, 2018.

$\sim$ " L'exercice de moyens de pression en cours de convention collective : I'impact de la liberté constitutionnelle d'association », Cahiers de Droit, 2018 (sous presse). 